\title{
Sub-millimeter $T_{2}$ weighted fMRI at $7 \mathrm{~T}$ : comparison of 3D-GRASE and 2D SE-EPI
}

\author{
Valentin G. Kemper ${ }^{1,2 *}$, Federico De Martino ${ }^{1,2,3}$, An T. Vu ${ }^{3}$, Benedikt A. Poser ${ }^{1,2}$, \\ David A. Feinberg ${ }^{4,5}$, Rainer Goebel ${ }^{1,2,6}$ and Essa Yacoub ${ }^{3}$ \\ ${ }^{1}$ Department of Cognitive Neuroscience, Faculty of Psychology and Neuroscience, Maastricht University, Maastricht, \\ Netherlands, ${ }^{2}$ Department of Cognitive Neuroscience, Maastricht Brain Imaging Center, Maastricht University, Maastricht, \\ Netherlands, ${ }^{3}$ Department of Radiology, Center for Magnetic Resonance Research, University of Minnesota Medical School, \\ Minneapolis, MN, USA, ${ }^{4}$ Advanced MRI Technologies, Sebastopol, CA, USA, ${ }^{5}$ Helen Wills Institute of Neuroscience, \\ University of California, Berkeley, Berkeley, CA, USA, ${ }^{6}$ Department of Neuroimaging and Neuromodeling, Netherlands \\ Institute for Neuroscience, Royal Netherlands Academy of Arts and Sciences (KNAW), Amsterdam, Netherlands
}

\section{OPEN ACCESS}

Edited by:

Nikolaus Weiskopf,

University College London, UK

Reviewed by:

Christian Windischberger, Medical University of Vienna, Austria Laurentius Huber, Max Planck Institute for Human Cognitive and Brain Sciences,

Germany

${ }^{*}$ Correspondence: Valentin G. Kemper,

Department of Cognitive Neuroscience, Faculty of Psychology and Neuroscience, Maastricht University, PO Box 616, 6200 MD Maastricht, Netherlands valentin.kemper@maastrichtuniversity.nl

Specialty section: This article was submitted to Brain Imaging Methods, a section of the journal Frontiers in Neuroscience

Received: 29 January 2015 Accepted: 20 April 2015 Published: 05 May 2015

Citation: Kemper VG, De Martino F, Vu AT, Poser BA, Feinberg DA, Goebel $R$ and Yacoub $E$ (2015) Sub-millimeter $T_{2}$ weighted $\mathrm{FMRI}$ at $7 \mathrm{~T}$ : comparison of 3D-GRASE and 2D SE-EPI.

Front. Neurosci. 9:163.

doi: 10.3389/fnins.2015.00163
Functional magnetic resonance imaging (fMRI) allows studying human brain function non-invasively up to the spatial resolution of cortical columns and layers. Most fMRI acquisitions rely on the blood oxygenation level dependent (BOLD) contrast employing $\mathrm{T}_{2}$ * weighted 2D multi-slice echo-planar imaging (EPI). At ultra-high magnetic field (i.e., $7 \mathrm{~T}$ and above), it has been shown experimentally and by simulation, that $\mathrm{T}_{2}$ weighted acquisitions yield a signal that is spatially more specific to the site of neuronal activity at the cost of functional sensitivity. This study compared two $\mathrm{T}_{2}$ weighted imaging sequences, inner-volume 3D Gradient-and-Spin-Echo (3D-GRASE) and 2D Spin-Echo EPI (SE-EPI), with evaluation of their imaging point-spread function (PSF), functional specificity, and functional sensitivity at sub-millimeter resolution. Simulations and measurements of the imaging PSF revealed that the strongest anisotropic blurring in 3D-GRASE (along the second phase-encoding direction) was about $60 \%$ higher than the strongest anisotropic blurring in 2D SE-EPI (along the phase-encoding direction). In a visual paradigm, the BOLD sensitivity of 3D-GRASE was found to be superior due to its higher temporal signal-to-noise ratio (tSNR). High resolution cortical depth profiles suggested that the contrast mechanisms are similar between the two sequences, however, 2D SE-EPI had a higher surface bias owing to the higher $\mathrm{T}_{2}{ }^{*}$ contribution of the longer in-plane EPI echo-train for full field of view compared to the reduced field of view of zoomed 3D-GRASE.

Keywords: 3D-GRASE, Spin-Echo EPI, T2, T2*, point-spread function, high-resolution BOLD fMRI

\section{Introduction}

Since its discovery more than two decades ago (Bandettini et al., 1992; Kwong et al., 1992; Ogawa et al., 1992), non-invasive functional magnetic resonance imaging (fMRI) has evolved to become a primary method to study human brain function. The majority of fMRI experiments have been conducted using the blood oxygenation level dependent (BOLD) method (Ogawa et al., 1990), relying on $\mathrm{T}_{2}{ }^{*}$ changes in gradient-echo based echo-planar imaging (GE-EPI, Mansfield, 1977) acquisitions. However, it has been shown 
in simulations (Boxerman et al., 1995; Uludag et al., 2009; Pflugfelder et al., 2011) and experiments at sub-millimeter (Duong et al., 2003; Yacoub et al., 2003, 2007) or lower resolution (Harmer et al., 2012; Boyacioglu et al., 2014; Panchuelo et al., 2015), that at high field strength (7 Tesla and above) imaging strategies utilizing $\mathrm{T}_{2}$ weighted contrast yield higher spatial specificity than $\mathrm{T}_{2}{ }^{*}$ weighted contrast. This becomes particularly relevant when the investigated functional organization is on the order of $1 \mathrm{~mm}$ or smaller, requiring avoidance of pial vein effects that have been shown to be stronger in $\mathrm{T}_{2}{ }^{*}$ weighted acquisitions (Yacoub et al., 2005). Cerebral blood flow or blood volume based approaches present an alternative approach to high-resolution fMRI (see e.g., Jin and Kim, 2008; Goense et al., 2012; Huber et al., 2015).

By far, most $T_{2}$ weighted fMRI experiments have been conducted using 2D spin-echo EPI sequences (Bandettini et al., 1994; see Norris, 2012 for review). The EPI echo train increases the temporal and the specific absorption rate (SAR) efficiency as compared to purely $\mathrm{T}_{2}$ weighted sequences such as RARE/FSE (Hennig et al., 1986; Constable et al., 1994; Poser and Norris, 2007) or to balanced and non-balanced steady-state free precession sequences (b-SSFP/nb-SSFP) (Auerbach et al., 2002; Bieri and Scheffler, 2007; Miller and Jezzard, 2008; Barth et al., 2010; Goa et al., 2014). However, the EPI echo train causes blurring in the phase-encoding direction due to $\mathrm{T}_{2}^{\prime}$ weighting (Constable and Gore, 1992) and increases the $\mathrm{T}_{2}{ }^{*}$ weighting as demonstrated by Goense and Logothetis (2006).

As an alternative, inner-volume excitation approaches, such as 3D Gradient-and-Spin-Echo (3D-GRASE) (Oshio and Feinberg, 1991; Feinberg et al., 2008) or single slice inner volume 2DSE EPI (Duong et al., 2002; Yacoub et al., 2003) have been proposed for $\mathrm{T}_{2}$ weighted sub-millimeter fMRI in humans. The 3D-GRASE sequence combines EPI-readouts with fast spinecho acquisition schemes (RARE/TSE) by acquiring multiple EPI readouts separated by refocusing pulses. Several secondary phase-encoding steps (referred to as "partitions" throughout this manuscript) are acquired after a single excitation pulse thus overcoming the single slice limitation of zoomed inner volume EPI. Slab-selective refocusing pulses are applied perpendicularly to the slab-selective excitation pulse to limit the required phase-encoding steps (Feinberg et al., 2008). This inner-volume selection allows for shorter EPI echo-trains, minimizing both $\mathrm{T}_{2}{ }^{*}$ contrast and blurring in the in-plane phase-encoding direction. At the same time, short EPI echo-trains keep the overall echo train length short to minimize blurring along the partition direction related to $T_{2}$ decay. Centric ordering of the partitions is typically chosen to sample k-space center at maximal SNR (minimal TE). Compared to 2D spin-echo EPI sequences, 3DGRASE offers a potential SAR advantage because it uses fewer radiofrequency $(\mathrm{RF})$ pulses due to the necessity of only one excitation pulse for the entire 3D volume and a reduced number of refocusing pulses due to partial Fourier acquisitions along the partition direction. The parsimony in SAR can be used to improve the excitation and refocusing slab profiles by increasing the bandwidth-time product of the RF pulses or to shorten the RF pulse duration in order to shorten the overall echo train length. Cortical depth-specific and columnar fMRI studies have been successfully conducted using 3D-GRASE (Zimmermann et al.,
2011; Olman et al., 2012), and it has been shown that 3D-GRASE yields a more specific signal than GE-EPI (De Martino et al., 2013). However, thus far, a systematic comparison of 3D-GRASE and 2D SE-EPI has been missing.

Therefore, this study compares 3D-GRASE and 2D SE-EPI for sub-millimeter resolution fMRI experiments at $7 \mathrm{~T}$. We do so by simulating and measuring the imaging point-spread function (referred as to PSF through this manuscript) reflecting the anisotropic spread of information in the image stemming from an idealized point source. The PSF is defined here as the magnitude of the Fourier transform of the complex optical transfer function, a function reflecting a modulation of kspace data (i.e., truncation associated with the finite nominal image resolution and signal reduction by relaxation weighting at different positions in $\mathrm{k}$-space), whose magnitude is the so-called modulation transfer function (MTF) (Robson et al., 1997). $\mathrm{T}_{2}$ or $\mathrm{T}_{2}{ }^{*}$ weighting along an echo-train and truncation of $\mathrm{k}$-space thus impose limits on the effective resolution of the image. Further, we measure temporal stability (temporal signal-to-noise ratio, tSNR), functional responses to visual stimulation, and the cortical depth profiles of the two acquisitions.

\section{Materials and Methods}

All measurements were performed on a 7T Siemens Magnetom scanner equipped with a body gradient system $(70 \mathrm{mT} / \mathrm{m}$ maximum amplitude, $200 \mathrm{mT} / \mathrm{m} / \mathrm{s}$ maximum slew rate) and a dedicated surface coil (16-channel receive and a separate four channel transmit coil with a pre-determined static B1 phase set optimized for homogeneity over the visual cortex; Live Services, Minneapolis, MN, USA) covering the posterior part of the subjects' heads. Compared to a whole brain coil, this RF coil offers better transmit efficiency (reduced SAR) in occipital areas and a wide angle for visual stimulation. Three subjects [two females, age $(28.3 \pm 1.5)$ years] were scanned for imaging PSF and functional measurements. Three additional male subjects (age $33.3 \pm 3.5$ ) were scanned for the PSF measurements only (see below). All subjects were scanned after giving informed written consent and in compliance with the local ethical board.

\section{Visual Stimulation Protocol}

Functional data were acquired while the subjects were performing a visual stimulation task. High contrast concentric flickering checkerboard patterns were displayed centrically on a screen in the magnet bore with a visual angle of $39^{\circ}$. A block design was chosen with 14 blocks of $10 \mathrm{~s}$ stimulation and $12 \mathrm{~s}$ rest. The subjects were instructed to fixate a point in the middle of the screen.

\section{Simulations}

The signal evolution throughout the echo trains of 3D-GRASE and 2D SE-EPI were simulated in MATLAB (The MATHWORKS Inc., Natick, MA, USA). The same imaging parameters were used in the PSF measurements as in the functional experiments (see below). For the 2D SE-EPI, the signal MTF, (Robson et al., 1997) was modeled as an exponential decay with a time constant $\left(\mathrm{T}_{2}\right)$ of $50 \mathrm{~ms}$ (see Uludag et al., 2009 for review of relaxation times) in addition to an exponential decay with a time constant 
$\left(\mathrm{T}_{2}^{\prime}\right)$ of $63 \mathrm{~ms}$ (where $\left.\mathrm{T}_{2}{ }^{*}=1 /\left(1 / \mathrm{T}_{2}+1 / \mathrm{T}_{2}^{\prime}\right)=27.8 \mathrm{~ms}\right)$ symmetrically on both sides of the central spin-echo (Bernstein et al., 2004), such that the outer k-space lines experience a stronger modulation. To account for partial Fourier acquisitions (Feinberg et al., 1986), the missing $\mathrm{k}$-space lines were zerofilled. Accelerated imaging was accounted for by assuming the acquisition of missing k-space lines interspersed equidistantly between the acquired lines such that the total echo-train duration was maintained.

The in-plane phase-encoding part of the 3D-GRASE sequence was simulated as in the 2D SE-EPI case, whereas the partition direction was simulated to experience an exponential decay with the same time constant $\left(\mathrm{T}_{2}=50 \mathrm{~ms}\right.$, equivalent to perfect $180^{\circ}$ refocusing pulses). Centric reordering was taken into account and the same procedure to mimic partial Fourier acquisition was applied.

In the model it was assumed that the modulation along the phase-encoding direction is separable from the modulation along the partition direction in 3D-GRASE. In order to obtain the simulated PSF at a $1 \mu \mathrm{m}$ resolution the simulated MTF was Fourier transformed after zero-filling to account for the truncated $\mathrm{k}$-space acquisition. This simulation was repeated for the phaseencoding direction of both sequences and the partition direction of 3D-GRASE. All parameter sets used in the experiments were considered. A normal distribution was fitted to the magnitude of each PSF using a linear least squares regression with four free parameters, amplitude, offset, standard deviation, and baseline. A Gaussian was chosen because it well approximated the shape of the central lobe of the PSF (i.e., the convolution of the decay filter and a sinc-kernel due to the k-space truncation) and it allows to easily parameterize the full width at half maximum (FWHM).

\section{Point-Spread Function Measurements}

PSF measurements were performed with the same imaging parameters as the functional measurements (see below) and the simulations, except for the number of measurements and a lower image scaling factor to adjust the dynamic range to accommodate the high image intensity in the k-space center. The vendor's product 2D SE-EPI sequence was used after implementing the option to turn off the phase-encoding gradients. Further, the gradient polarity of the refocussing gradient was inverted to allow low-SAR fat suppression (Park et al., 1987; Volk et al., 1987; Gomori et al., 1988; Ivanov et al., 2010) in the PSF measurements and the functional measurements.

Four volumes were acquired to reach a steady state. In the fifth repetition, the phase-encoding gradients were switched off, so that the $\mathrm{k}$-space center was acquired in every readout line. This procedure yields non-Fourier-encoded images (Fourierencoded only along the readout direction), which are processed in the image reconstruction pipeline nonetheless. Since the acquired data does not contain any spatial information, but does experience the MTF (i.e., the weighting due $T_{2}$ and $T_{2}{ }^{*}$ decay), the resulting image reflects the PSF due to the acquisition and reconstruction (Mugler et al., 1992; Robson et al., 1997; Zaitsev et al., 2004; Park et al., 2007). In the ideal case with no signal decay, all acquisition lines would be identical leading to a reconstructed image with a delta-function like intensity distribution along the non-encoded directions. However, in practice, due to the non-uniformity of the MTF, the peak has a finite width and exhibits side lobes in the case of partial Fourier acquisitions.

The data were analyzed as follows. After standard image reconstruction, individual lines (in phase-encoding or partition encoding direction) were admitted to the analysis if their maximum pixel value was at least $25 \%$ of the global image intensity to avoid noise-only lines. These lines were centered on their respective maximums. Then, data were interpolated using a spline interpolation and a normal distribution was fitted as to the simulated data described above. In the 2D SE-EPI acquisitions, as many peaks as the GRAPPA acceleration factor appear in the data due to the aliasing, but only the maximum peak was used for fitting. This makes the results more comparable to the 3D-GRASE data and reduces an overestimation of the PSF due to the collapsing of the PSF over the entire FoV (see Discussion for details).

In order to assess the slice profile of 2D SE-EPI (i.e., the point spread function along the slice direction), we simulated profiles of the used excitation and refocusing pulses by Fourier transforming the vendor-provided RF pulse shapes. Perfect 90 and $180^{\circ}$ pulses were assumed at the slice center. The resulting slice profiles were calculated as $\sin \left(\alpha_{1}\right) \times \sin ^{2}\left(\alpha_{2} / 2\right)$, where $\alpha_{1}$ and $\alpha_{2}$ denote the spatially dependent excitation and refocusing angles, respectively. The final slice profile was obtained by assuming saturation recovery from the excitation pulse during one TR at the original slice location and during half a TR at the location of the neighboring slices (approximating interleaved slice acquisition). Saturation recovery from both a fully relaxed system (longitudinal magnetization $\mathrm{m}_{\mathrm{z}}=1$ ) and an Ernst steady state were considered in a range of $T_{1}$ values between 1200 and $1800 \mathrm{~ms}$ (step size of $100 \mathrm{~ms}$ ).

\section{Functional Measurements}

Five different acquisitions were repeated two times in each scanning session in a pseudo-random order. All acquisitions had a nominal isotropic resolution of $(0.8 \mathrm{~mm})^{3}$ of and repetition time (TR) of $2000 \mathrm{~ms}$. All used nominal excitation/refocussing flip angles of $90 / 180^{\circ}$. Additional acquisition parameters are listed in Table 1. 3D-GRASE 2 is identical to 3D-GRASE 1 except the image volume was rotated about the read-direction such that the phase-encoding direction of 3D-GRASE 1 was the same as partition encoding direction of 3D-GRASE 2 and vice versa. The slices in all measurements except 3D-GRASE 2 were placed parallel to calcarine sulcus (anterior-posterior, ant-pos), and orthogonally to that in 3D-GRASE 2 (inferior-superior, inf-sup). Phase-encoding direction left-right was chosen for all 2D SE-EPI acquisitions to keep the FoV to a minimum, while avoiding aliasing in the region of interest. Although the echo spacing of the 2D SE-EPI acquisitions had to be slightly increased with respect to 3D-GRASE to avoid critical peripheral nerve stimulation, phase-encoding direction left-right allowed much shorter EPI echo train durations than anterior-posterior in 2D SE-EPI.

Automatic second order shimming routines were used to homogenize the static magnetic field (B0) in a region of interest big enough to accommodate the 2D SE-EPI FoV. The shim was adjusted manually for further improvements of 
TABLE 1 | Acquisition parameters of the five functional imaging protocols.

\begin{tabular}{|c|c|c|c|c|c|}
\hline & 3D-GRASE 1 & 3D-GRASE 2 & 2D SE-EPI 1 & 2D SE-EPI 2 & 2D SE-EPI 3 \\
\hline TE (ms) & 36 & 36 & 36 & 50 & 50 \\
\hline Field of view & $30 \times 120 \times 10$ & $30 \times 120 \times 10$ & $120 \times 120 \times 16$ & $120 \times 120 \times 16$ & $120 \times 120 \times 16$ \\
\hline Echo spacing (ms) & 1.01 & 1.01 & 1.02 & 1.02 & 1.03 \\
\hline EPl/total echo train duration (ms) & $30 / 216$ & $30 / 216$ & 46 & 46 & 31 \\
\hline GRAPPA acceleration factor & - & - & 2 & 2 & 3 \\
\hline Partial Fourier & 5/8 (partition direction) & 5/8 (partition direction) & 6/8 (phase direction) & 6/8 (phase direction) & 6/8 (phase direction) \\
\hline Slice orientation & ant-pos & inf-sup & ant-pos & ant-pos & ant-pos \\
\hline
\end{tabular}

the homogeneity, until the FWHM of the measured Larmor frequencies was well below $40 \mathrm{~Hz}$. Macroscopic inhomogeneities further cause a reduction in the apparent $\mathrm{T}_{2}{ }^{*}$. We obtained a macroscopic, volumetric $\mathrm{T}_{2}{ }^{*}$ of $17 \pm 2 \mathrm{~ms}$ (mean $\pm \mathrm{SD}$ across all six participants). The individual subjects' volumetric $\mathrm{T}_{2}{ }^{*}$ values were also used in the PSF simulations in addition to the theoretical $\mathrm{T}_{2}{ }^{*}$ values for cortical gray matter. A constant shim was then used throughout all functional and PSF measurements. The transmit field $(\mathrm{B} 1+)$ was measured using a pre-saturation based B1-mapping sequence provided by the scanner vendor. The transmit voltage was adjusted to yield the correct flip angles in the approximate location of the calcarine sulcus.

\section{Anatomical Measurements}

Structural images were acquired for anatomical reference using a $\mathrm{T}_{1}$ weighted MP-RAGE sequence (isotropic resolution of $0.6 \mathrm{~mm}$; $\mathrm{TR}=3100 \mathrm{~ms}$; $\mathrm{TI}=1500 \mathrm{~ms} ; \mathrm{TE}=2.52 \mathrm{~ms}$; flip angle $=5^{\circ}$; GRAPPA acceleration factor $=3 ; \mathrm{FoV}=230 \times 230 \times 154 \mathrm{~mm}^{3}$ ). Additional proton density weighted images were acquired using identical imaging parameters (except TR $=1440 \mathrm{~ms}$ ) without the inversion module. These additional images were used to alleviate inhomogeneities across the FoV in post-processing by dividing the $\mathrm{T}_{1}$ weighted image pixel values by those of the proton density weighted images (van de Moortele et al., 2009).

\section{Functional Data Analysis}

Data analysis was performed using BrainVoyager QX 2.8 (Brain Innovation, Maastricht, The Netherlands) and custom-written routines in MATLAB. Standard preprocessing of the fMRI data consisted of $3 \mathrm{D}$ rigid body motion correction and temporal high-pass filtering by a General Linear Model (GLM) with three cosine cycles and a linear trend regressor for each run. The average motion parameters for all acquisitions were $0.32 \pm$ $0.31 \mathrm{~mm}$ translation and $0.31 \pm 0.28$ degree rotation (mean \pm $\mathrm{SD}$ of absolute deviation of both parameters). No difference between 3D-GRASE and 2D SE-EPI acquisitions was observed. SE-EPI data was additionally slice-time corrected using a sincweighted interpolation. These data were then co-registered with the structural images using positional information of the acquisitions. Manual adjustments were performed using edge information in the fMRI data. For this purpose, the fMRI data was averaged across time after preprocessing to yield a single high quality image, with fine-grained structural information (i.e.,
CSF/gray matter and white matter/gray matter boundaries), for alignment. When image distortions were too strong to guarantee good alignment of the entire volume, multiple alignments were performed specifically in order to optimize the co-registration for the targeted regions, in which cortical depth profiles were generated. Finally, functional images were resampled in the $3 \mathrm{D}$ anatomical volume at a resolution of $0.8 \mathrm{~mm}$ using a sinc interpolation.

A standard GLM was used to assess the functional activation. The visual stimulation predictor was created by convolving the visual activation blocks with a standard hemodynamic response function (HRF). For each imaging sequence, both runs were analyzed together on a single subject level without additional confound predictors.

tSNR maps were created by dividing the voxelwise temporal mean intensity by the temporal standard deviation. To produce tSNR maps, preprocessing included only high-pass filtering, using four cosine cycles and linear trend removal. No motion correction or slice-time correction was applied to avoid additional spatial interpolation. The effect of the visual stimulation was removed from the voxels' time course using univariate regression (i.e., GLM).

For the cortical depth profile analysis, high resolution cortical depth grids were created similar to as described in De Martino et al. (2013) based on anatomical data after segmenting cortical gray and white matter and delineating the boundary between gray matter and cerebrospinal fluid (CSF). The automatic segmentation in BrainVoyager QX was used and refined manually in the regions of interest to ensure correct classification of white matter, gray matter and other tissue types. In each region of interest, a stack of five cortical grids were created starting from cortical thickness measurements (Jones et al., 2000). Grids were placed at regular intervals of cortical depth, and neighboring sampling points within a grid were regularly spaced. A single grid would sample $8 \times 8 \mathrm{~mm}^{2}$ on a flat piece of cortex. A total of 10 regions of interest were defined within the foldings of the calcarine sulcus. Figure 1 displays an exemplary cortical depth sampling region of interest.

After the creation of relative cortical depth grids, the local cortex normal vectors (the vectors orthogonal to the cortical sheet) were approximated linearly by calculating the difference vector between the grid points at $10 \%$ cortical depth and the corresponding grid points at $90 \%$ cortical depth. The angle between these cortex normal vectors and the orientation of the 


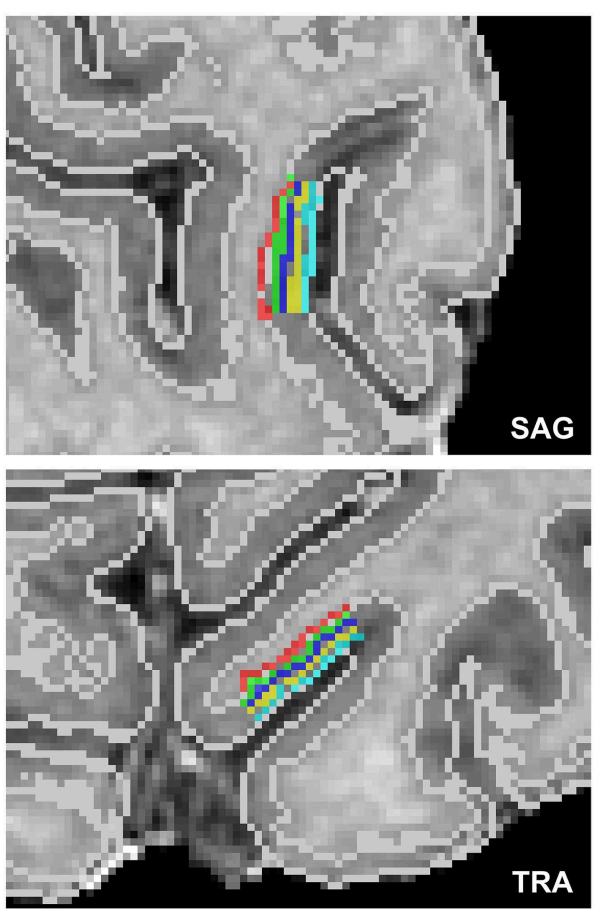

FIGURE 1 | Exemplary high resolution cortical grid sampling prescription. Cortical depth dependent grids were prescribed at $10 \%$ (red), $30 \%$ (green), 50\% (dark blue), 70\% (yellow), and 90\% (light blue) cortical depth as defined between the white matter-gray matter boundary and the gray matter-CSF boundary (white lines), as segmented based on the anatomical reference images.

partition directions of both 3D-GRASE 1 and 3D-GRASE 2 were calculated.

\section{Results}

\section{Imaging Point-Spread Function Simulations and Measurements}

Figure 2 shows the results of the simulations and the imaging PSF measurement for all acquisitions, demonstrating good agreement between experimental data and simulations for both the MTF (top) and the PSF (bottom). Prior to the creation of the MTFs, the raw data from the PSF measurements were coil-combined (sum-of-squares combination). The maximum of each readout line (i.e., the echo) was selected and averaged across the other imaging dimension (slices/partition direction for phase-encoding direction; phase-encoding direction for partition direction), and normalized in the range $[0,1]$. Note the asymmetry in MTF with higher intensities in the echoes before than after the central spin-echo as expected from combined $\mathrm{T}_{2}$ and $\mathrm{T}_{2}^{\prime}$ modulation. This signal characteristic is often neglected in over-simplified models assuming only a symmetric $\mathrm{T}_{2}{ }^{*}$ signal decay around the spin-echo.

Table 2 reports the mean across lines and subjects of the full width half maximum of the PSF (mean \pm standard deviation). The FWHM of the partition direction in 3D-GRASE 1 was found to be $3.6 \pm 0.5$ pixels, which is about 56 and $63 \%$ higher than the FWHMs of the phase-encoding direction of 2D SE-EPI 1 and 2. Phase-encoding direction of 2D SE-EPI 3 and 3D-GRASE 1 acquisitions have smaller FWHMs due to the reduced number of phase-encoding steps. The experimental findings are in good agreement with the simulations. The agreement is enhanced when using the experimentally obtained volumetric $\mathrm{T}_{2}{ }^{*}$ values (right column) in the simulation rather than literature values for gray matter (middle column).

The simulation results of the 2D SE-EPI slice profile are displayed in Figure 3. The FWHM (indicated by the vertical dashed line in the figure) was found to be $1.3 \pm 0.2$ times the nominal slice thickness (mean $\pm \mathrm{SD}$ across different $\mathrm{T}_{1}$ values and saturation scenarios). Due to the wide saturation bands of neighboring slices, the effective slice profile resembles a triangle rather than the desired rectangle. When taking into account the saturation bands of neighboring slices, the integral of the attainable signal is reduced by $49 \pm 5$ percent compared to a single slice without $\mathrm{T}_{1}$ saturation effects and by $35 \pm 5$ percent compared to a single slice with $\mathrm{T}_{1}$ saturation effects.

\section{Temporal Signal-to-Noise Ratio}

Figure 4 displays central slices of the tSNR maps and full volume tSNR histograms. The 2D SE-EPI data were cropped to the same in-plane FoV as the 3D-GRASE 1 acquisition (disregarding increased image distortions in 2D SE-EPI). The images and histograms show that the tSNR is substantially higher in 3DGRASE than in all 2D SE-EPI acquisitions. 3D-GRASE 2 yields similar tSNR as 3D-GRASE 1 (not shown because of its different slice orientation). The 2D SE-EPI 1,2 , and 3 [short TE $(R=2)$, long TE $(R=2)$, long TE $(R=3)]$ yield tSNR in descending order as expected due to $\mathrm{T}_{2}$ relaxation, undersampling $(R)$, and noise amplification (g-factor) from higher acceleration. Similar results were found for all other subjects (not shown).

\section{Visual Activation}

All functional acquisitions elicited significant responses to the visual stimulation in and around the calcarine sulcus. Figure 5 displays results of the functional experiment overlaid on both an anatomical reference (sagittal cut, left), and on averaged functional slices (single central slice). The same statistical threshold was chosen for all acquisitions, and no multiple comparison correction was used in order not to favor the reduced FoV acquisitions. Note the absence of activated voxels in white matter regions in all acquisitions. BOLD sensitivity is significantly lower in the $2 \mathrm{D}$ SE-EPI acquisitions as a consequence of the reduced tSNR (see Figure 4).

Figure 6 displays histograms of the BOLD signal changes (top) and $t$-values (bottom) for all three subjects. For each acquisition, voxels were included in the analysis if they responded significantly ( $p<0.05$ uncorrected). Only the mutually covered region was considered. Border voxels were discarded to exclude artifacts from motion or slow scanner drift. The histograms reveal that in each subject, smaller BOLD signal changes surpass the significance threshold using 3D-GRASE, indicative of smaller variability in the responses. In consequence, more active voxels 

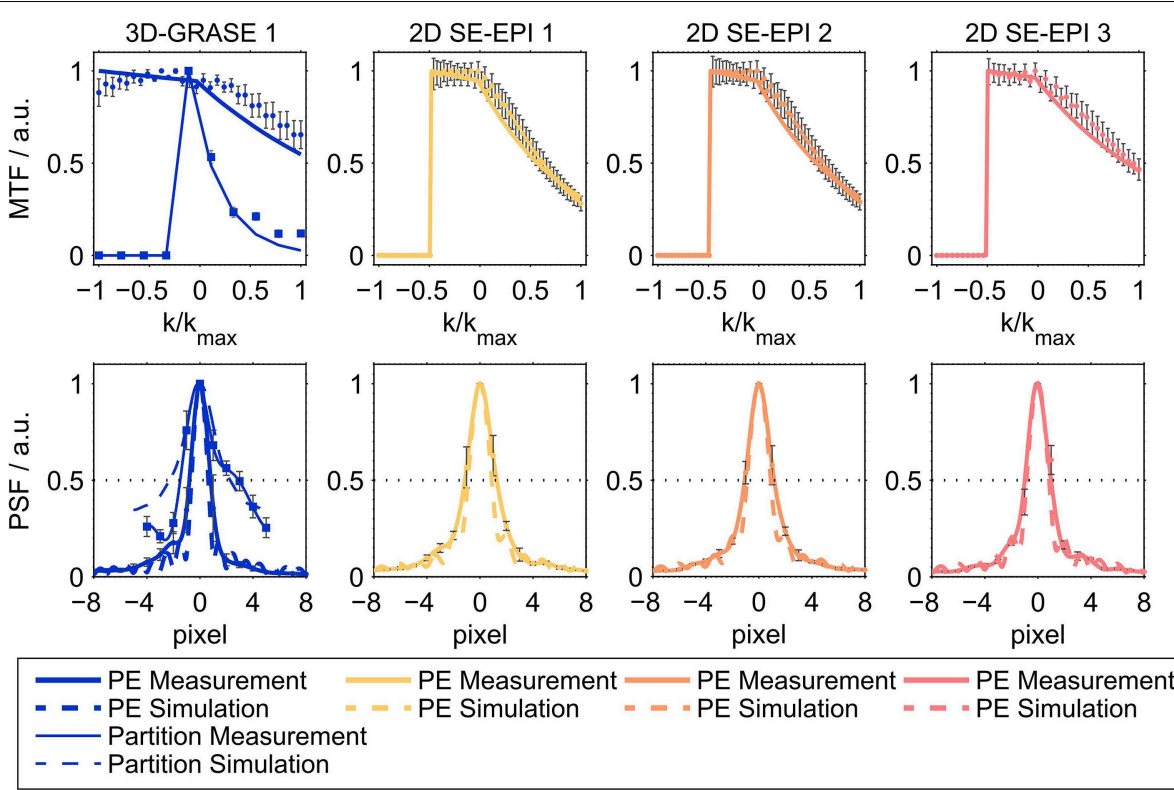

FIGURE 2 | Averaged modulation transfer functions (MTFs, top) and point-spread functions (PSFs, bottom). The top panel shows averaged MTFs in phase-encoding direction (thick lines) and partition encoding direction for 3D-GRASE sequences (thin lines). Solid lines represent averaged experimental results across all subjects, dashed lines represent simulations. The dimension of the horizontal axis in the top panel is relative $\mathrm{k}$-space. In the bottom panel, the dimension of the horizontal axis is pixels in absolute numbers (cropped to center) for better comparison. Error bars indicate standard deviation across subjects.
TABLE 2 | FWHM of the PSFs of the different acquisitions (mean \pm standard deviation).

\begin{tabular}{llccc}
\hline Acquisition & Direction & \multicolumn{3}{c}{ FWHM (pixel) } \\
\cline { 3 - 5 } & & Measurement & $\begin{array}{c}\text { Simulation } \\
\text { GM T 2 }^{*}\end{array}$ & $\begin{array}{c}\text { Simulation } \\
\text { Exp. . 2 }\end{array}$ \\
\hline 3D-GRASE 1 & PE & $1.8 \pm 0.3$ & 1.2 & $1.3 \pm 0.0$ \\
2D SE-EPI 1 & PE & $2.3 \pm 0.2$ & 2.0 & $2.3 \pm 0.1$ \\
2D SE-EPI 2 & PE & $2.2 \pm 0.1$ & 2.0 & $2.3 \pm 0.1$ \\
2D SE-EPI 3 & PE & $2.0 \pm 0.1$ & 1.8 & $2.0 \pm 0.1$ \\
3D-GRASE 1 & partition & $3.6 \pm 0.5$ & 4.4 & \\
\hline
\end{tabular}

are detected in 3D-GRASE 1 than in any of the 2D SE-EPI acquisitions (see Figure 6 bottom).

\section{Cortical Depth Profiles}

Figure 7 displays cortical depth profiles from individual regions and their average from 3D-GRASE 1, 2D SE-EPI 1, and 2D SEEPI 3. 2D SE-EPI 2 was similar to 2D SE-EPI 1 and 2D-SE-EPI 3 (not shown for clarity). Grid points were included in the spatial average, if at least one of the three displayed acquisitions elicited significant responses ( $p<0.05$, uncorrected) in at least one of the five cortical depths. The responses measured with these protocols are similar showing BOLD signal changes mostly between 1 and $3 \%$. In both 2D SE-EPI and 3D-GRASE the BOLD signal change is significantly higher at $50 \%$ cortical depth than at $10 \%$ (twosided Wilcoxon signed-rank test, $p<0.02$, uncorrected). The

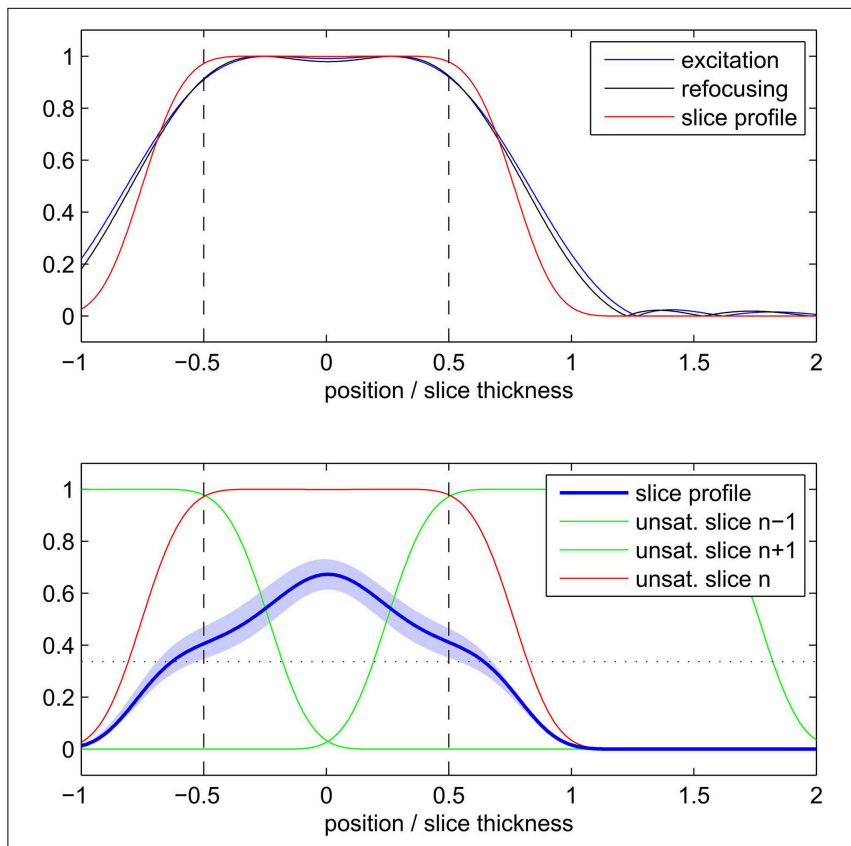

FIGURE 3 | Simulated 2D SE-EPI slice profile of a single slice (top) and with consideration of neighboring slices and saturation recovery (bottom). Profiles are displayed relative to nominal slice thickness.

2D SE-EPI acquisitions increase further toward the pial surface (signal change is different between 50 and $90 \%$ at $p<0.02$, two-sided Wilcoxon signed-rank test, for 2D SE-EPI 1 and 2, 


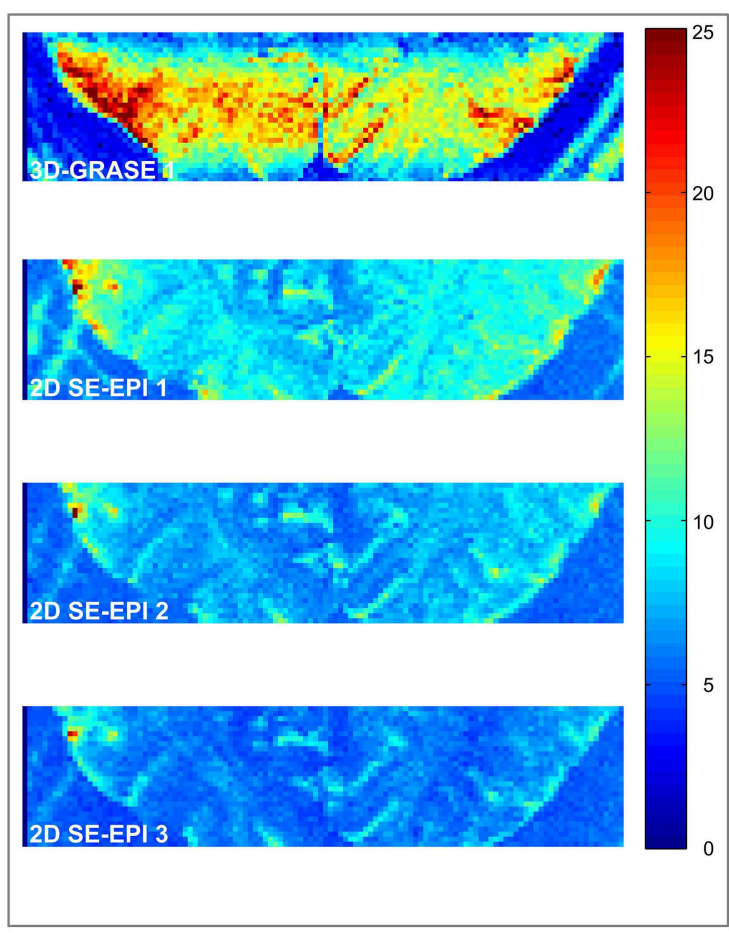

FIGURE 4 | Temporal signal-to-noise-ratio maps. Maps of the five different acquisitions in a single subject (left) and corresponding histograms (right) are displayed. The 2D SE-EPI images are cropped to the same FoV
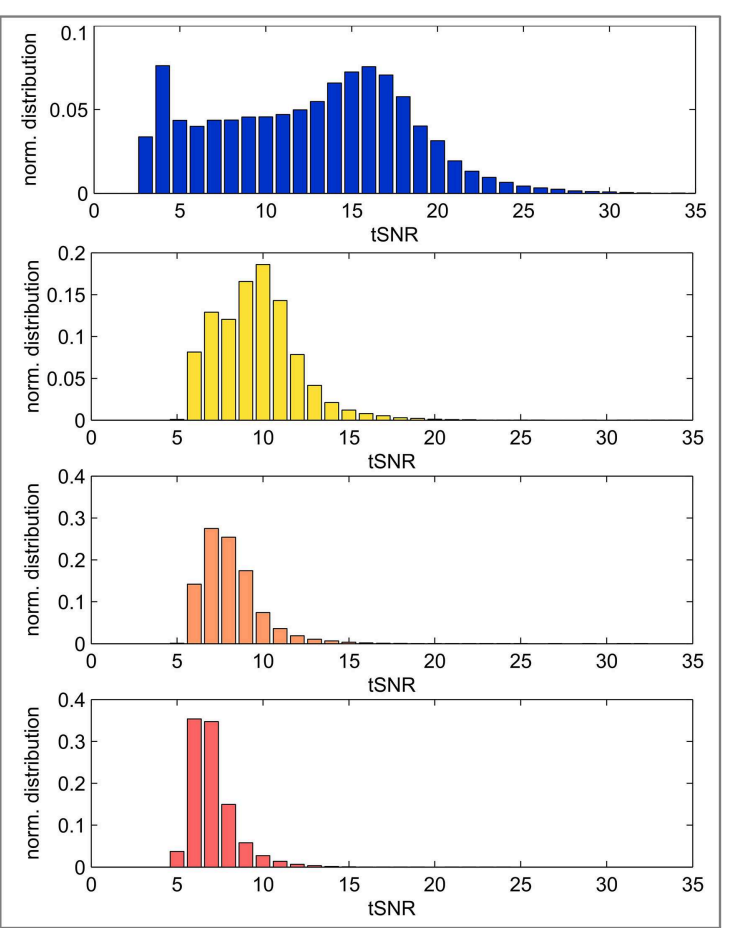

as 3D-GRASE 1. All maps have the same color scale. Histograms display the tSNR distribution of the same in-plane FoV in all 3D-GRASE slices and 2D SE-EPI slices corresponding to 3D-GRASE 1. not significant for 2D SE-EPI 3), whereas the 3D-GRASE profile shows a trend to decrease toward the surface.

In order to assess the potential effect of the imaging PSF on the cortical depth profiles dependent profiles, we repeated the cortical depth profile analysis after smoothing the 2D SE-EPI 1 data using an anisotropic Gaussian kernel. We used two different sizes of the smoothing kernel (FWHM of 2.4 and 4.7 pixels) in slice direction (no additional smoothing in other directions) a range that includes the estimated FWHM of 3D-GRASE 1 in the partition direction. The comparison of normal and blurred data is shown in Figure 8. The noted increase toward the pial surface in 2D SE EPI remains despite the smoothing. All profiles differ significantly between 10 and $90 \%$ cortical depth (two-sided Wilcoxon signed-rank test, $p<0.002$, uncorrected).

We also evaluated the difference between 3D-GRASE 1 and the orthogonally oriented 3D-GRASE 2 in four of the individual regions which were sufficiently covered by both acquisitions (ROIs 2, 5, 7, and 10 in Figure 7). Histograms of the angle (limited between 0 and $90^{\circ}$ ) between the cortex normal vectors and the partition directions of both 3D-GRASE 1 and 2, are presented at the top of the Figure 9. The averages of the cortical depth profiles in these regions are displayed at the bottom in the same figure. Despite the difference in acquisition orientation the depth dependent functional profiles are similar to one another, and are similar to the 3D-GRASE 1 profile in Figure 9 although only a subset of ROIs was used.

\section{Discussion}

In this study, we compared 3D-GRASE to 2D SE-EPI acquisitions in a high spatial resolution BOLD fMRI experiment at $7 \mathrm{~T}$. Simulations, PSF measurements and a simple visual activation paradigm with robust functional responses were used to assess differences with regard to voxel blurring, functional sensitivity, and functional (BOLD) specificity as indicated by cortical depth dependent profiles. Our findings suggest that the anisotropic blurring in 3D-GRASE is higher than in 2D SE-EPI. Further, 3D-GRASE yields a more sensitive BOLD signal and less macrovascular weighting as indicated by the smaller superficial effect in the cortical depth profiles. Based on these findings, we conclude that with the used experimental set-up 3DGRASE yields valuable advantages over 2D SE-EPI for highresolution $\mathrm{fMRI}$ applications provided the more limited coverage is sufficient to the experimental setup. The ability to discriminate small, differential signals at a length-scale on the order of $1 \mathrm{~mm}$ depends on the product of tSNR and effect-size (Murphy et al., 2007), which is proportional to the contrast-to-noise ratio (CNR). The measured effect-size (i.e., difference in BOLD signal) depends on the image blurring, and hence depends on the orientation of the structure with respect to the anisotropic image blurring. It can be shown that, for the protocols employed here, the superior tSNR of 3D-GRASE outweighs detrimental effects of the stronger image blurring, and that the CNR will be below that 


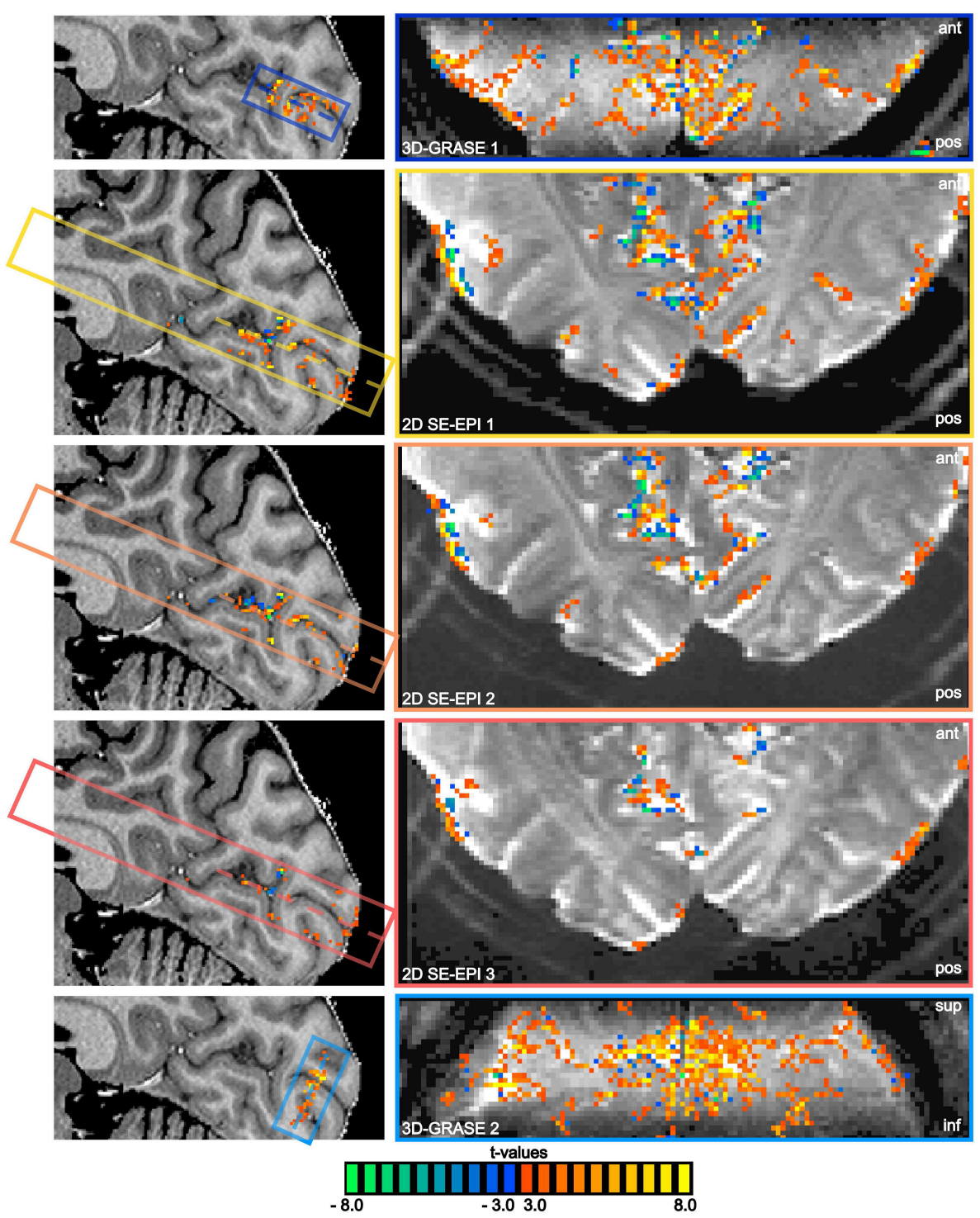

FIGURE 5 | BOLD responses overlaid on anatomical reference and averaged functional slices. Mid-sagittal slices of the reference anatomical scan (left) and central functional slices (right) are shown, as indicated by the dashed lines. Note the oblique coronal orientation of 3D-GRASE 2. The same statistical threshold was applied to all acquisitions $(t>3.0, p<0.003$ two-sided $t$-test on single voxel level, cluster threshold of 4 voxels). The position of the sagittal cut is shown on a transversal slice in the bottom left corner. The extent and orientation of the functional slices as well as the displayed slice position (dashed lines) are indicated by the colored boxes in the sagittal images. of 2D SE-EPI only if the investigated structure is closely aligned $\left(\sim 0^{\circ}\right)$ with the partition direction of $3 \mathrm{D}$-GRASE.

Our results are in general agreement with previous studies investigating 3D-GRASE (De Martino et al., 2013) or 2D SEEPI (Goense and Logothetis, 2006; Harel et al., 2006; Jin and Kim, 2008), independently. The different protocols we used followed practical considerations and were performed similarly to previously published studies while also matching parameters such as resolution and echo time. Compared to previous studies, the imaging FoV of the 3D-GRASE acquisitions was reduced to account for the slower switching of the body gradient coil (SC72) that was used here.
Inner-volume selection was previously proposed to reduce the echo train length in 2D SE-EPI using spatially orthogonal refocusing pulses to acquire a single slice (Yacoub et al., 2007, 2008). Multi-slice acquisitions with this approach are suboptimal because of saturation effects. Outer-volume suppression (OVS, Heidemann et al., 2012) and 2D spatially selective RF pulses (Pauly et al., 1989; Finsterbusch, 2010, 2013) have also been proposed. However, magnetization transfer effects in OVS would reduce the tSNR of 2D SE-EPI further, while additional RF-pulses would tighten the SAR constraints (Pfeuffer et al., 2002; Wargo and Gore, 2013) leading to a reduced number of slices. The use of segmented acquisitions would require techniques to 

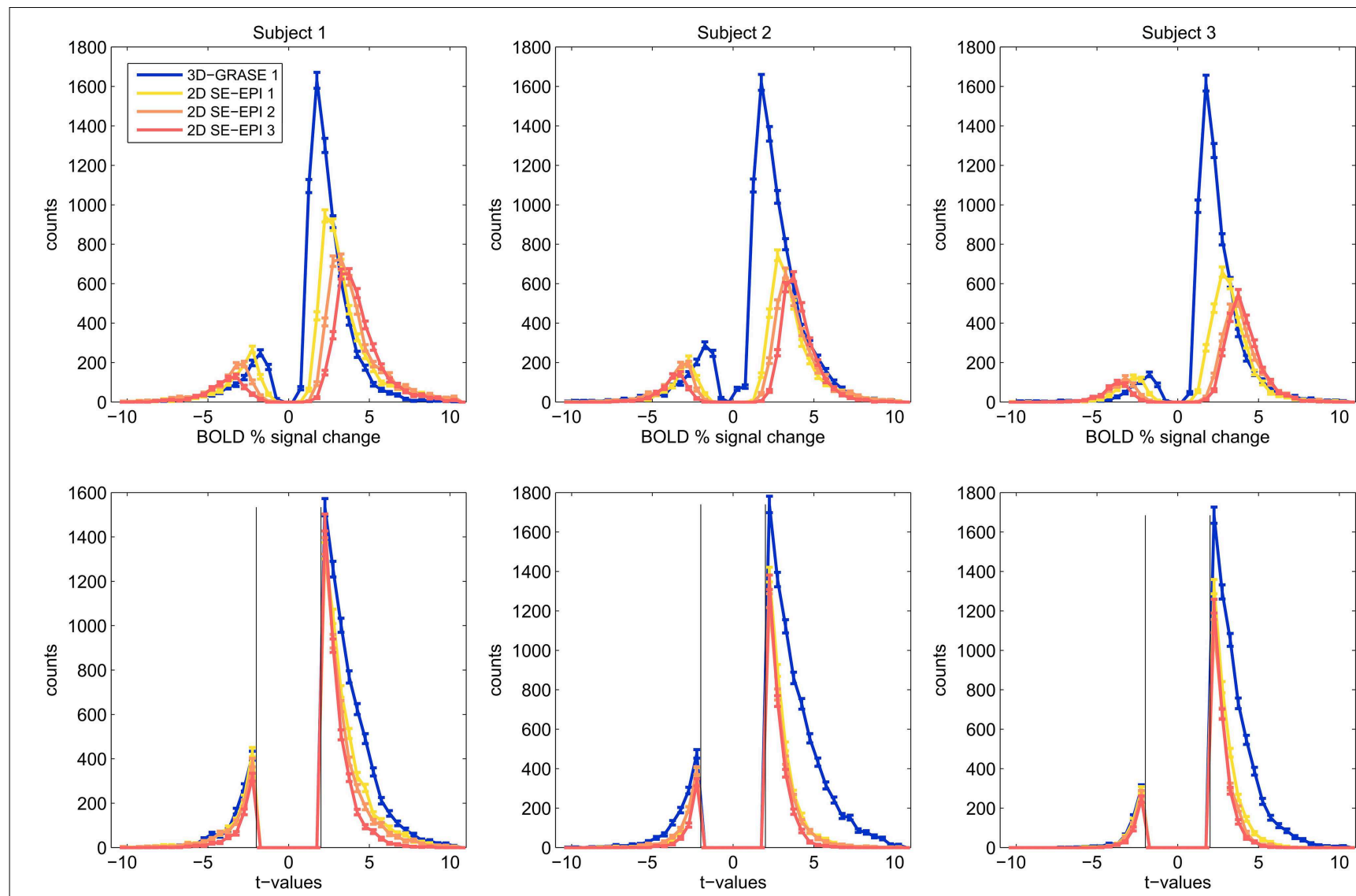

FIGURE 6 | Histograms of significant voxels. Counts of BOLD signal changes (top) and $t$-values are shown for voxels, which responded significantly to the stimulation $(t>2.0, p<0.046)$.

mitigate the effects of subject motion (e.g., the use of bite-bars or advanced image reconstruction techniques) and would be significantly less time efficient. Finally, a surface receive coil with an even more limited FoV (Koning et al., 2013) was not available to us and may represent an alternative way to achieve zoomed imaging in 2D multi-slice imaging.

\section{Imaging Point-Spread Function}

3D-GRASE was found to exhibit a wider PSF in partition direction than the 2D SE-EPI acquisitions in phase-encoding direction or slice direction (as expected from simulations). The blurring in the phase-encoding direction of 3D-GRASE was minimal because of the short EPI echo train duration due to the reduced FoV.

The PSF was assessed based on non-phase-encoded data from the entire FoV. Two concomitant sources of possible confounds should be taken into consideration. First, different spatial locations have different PSF properties due to the relaxation properties of their underlying anatomical tissue. Since $T_{2}$ of white matter and gray matter is almost identical at $7 \mathrm{~T}$ (Yacoub et al., 2003; Deistung et al., 2008; Cox and Gowland, 2010) and $\mathrm{T}_{2}{ }^{*}$ of white matter is only slightly shorter (Peters et al., 2007; Deistung et al., 2008), one can expect this influence to be small. CSF, which has a much longer $\mathrm{T}_{2}$, occupies only a small volume fraction in the chosen FoV and is partly saturated due to its long $\mathrm{T}_{1}\left(\mathrm{~T}_{1} \sim \mathrm{TR}\right)$. Other tissue compartments, which have much shorter $\mathrm{T}_{2}$, contribute relatively little to the signal at TE. It can therefore be expected that the presence of various tissue types does not significantly affect the PSF estimation. Second, static field inhomogeneities across the FoV lead to an overestimation of the global PSF by a broadening of the measured signal. Local off-resonances shift the voxel-by-voxel peak along the phaseencoding direction. The sum of the shifted peaks is broader than an individual one would be. Also, the effective volumetric $\mathrm{T}_{2}{ }^{*}$ is shortened. Either of these effects (tissue or field specific inhomogeneities) would lead to an overestimation or larger PSF. As such, our measurements can be regarded as a worst-case approximation of the local blurring. The simulations using gray matter $\mathrm{T}_{2}{ }^{*}$, on the other hand, reflect the ideal case and can be seen as a lower bound of the local blurring, not subject to either experimental imperfections affecting the local blurring or the global overestimation affecting only our measurements. The reasonable agreement of these numbers suggests that the detrimental effects of the global estimation are limited. An alternative approach, circumventing these possible confounds, would be to employ an acquisition scheme similar to the one by (Zeng and Constable, 2002; Chung et al., 2011), which was used for distortion correction in EPI images. In this study, we chose an 

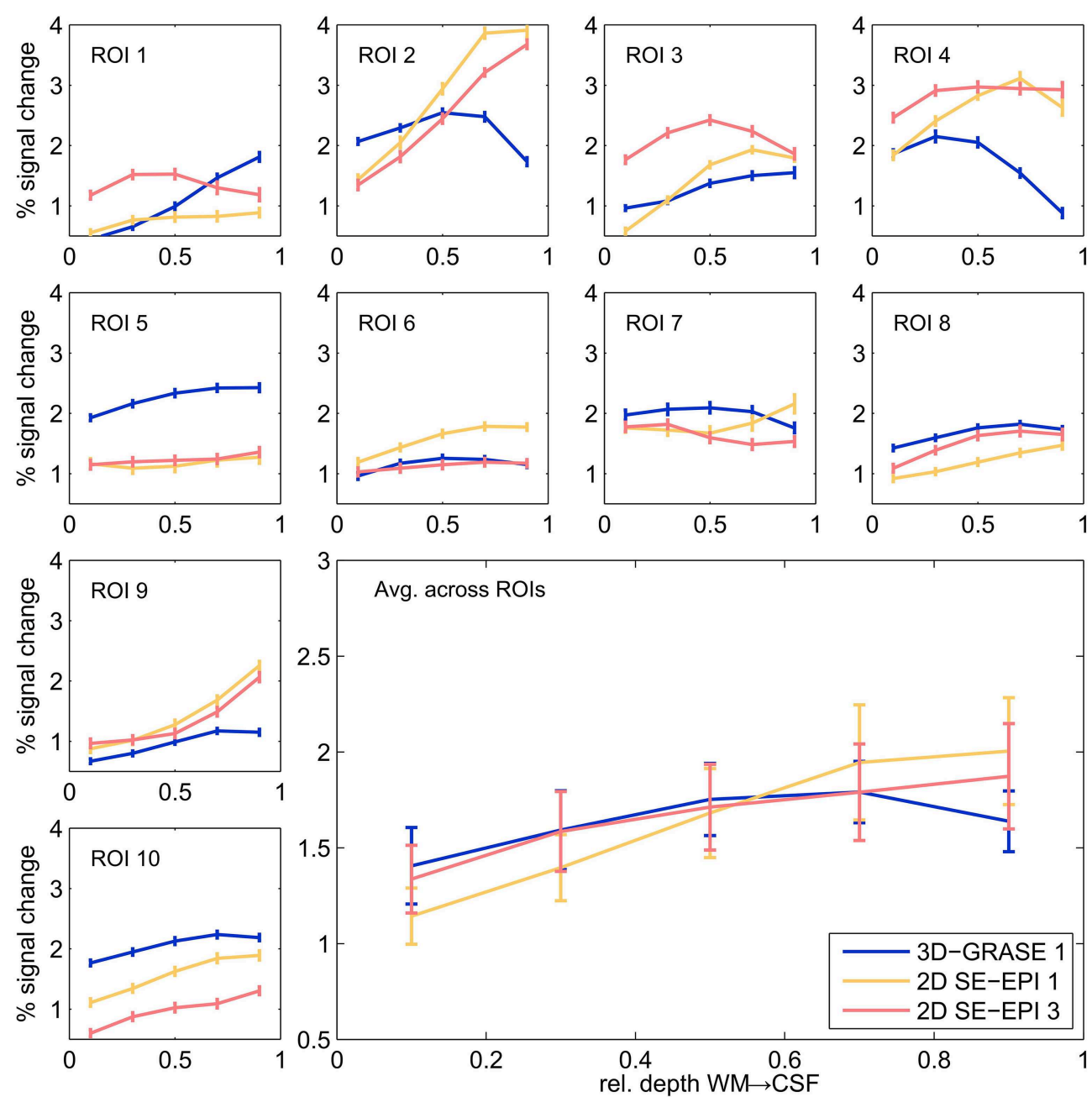

FIGURE 7 | Cortical depth profiles in individual regions. Single regions (small graphs) and their average (large graph, bottom right) of 3D-GRASE 1 (blue), 2D SE-EPI 1 (yellow), and 2D SE-EPI 3 (pink). Error bars represent standard errors across grid points (individual ROI graphs) and across regions (average graph).

approach that did not depend on image reconstruction, which is desirable when comparing different imaging pulse sequences.

This work focused on imaging related effects on the PSF and we did not consider the additional effect of the BOLD related spatial spread of the signal (i.e., the functional PSF), both of which need to be accounted for in determining the ultimate resolution of the fMRI signals. The latter is normally measured by considering differential paradigms with a known spatial distribution of responses and comparing it to the measured spatial distribution (Shmuel et al., 2007). In light of the results presented here, it is worth noting that a wider spatial imaging PSF will not cancel out high spatial frequency information in differential fMRI paradigms. Rather, when adjacent areas respond to different functional conditions, a wider spatial imaging PSF has a similar effect as a wider functional PSF and results in a reduction of CNR between the functional conditions (Yacoub et al., 2008). Thus, the combination of a differential fMRI paradigm, along with a high functional CNR with reduced contamination from pial veins, explain why columnar or cortical depth dependent fMRI analyses (Zimmermann et al., 2011; Olman et al., 2012; De Martino et al., 2013), with blurring levels (in 3D GRASE acquisitions) comparable to the data presented here, are possible.

\section{tSNR and BOLD Sensitivity}

The sensitivity to detect small, significant BOLD changes was found to be clearly superior in 3D-GRASE in this study (Figures 5, 6). This is a straight-forward consequence of the higher tSNR (Figure 4). The tSNR advantage of 3D-GRASE is likely to stem from two sources: First, being a 3D sequence 3DGRASE is more SNR efficient because of the longer effective sampling time per volume (Edelstein et al., 1986). This is particularly relevant since such high-resolution fMRI typically operates in the thermally dominated noise regime (Triantafyllou et al., 2005), in which tSNR scales linearly with SNR. Second, the inner volume selection in 3D-GRASE obviated the need for parallel imaging in 3D-GRASE, whereas it was necessary in 2D SE-EPI causing a loss in static SNR and hence tSNR 

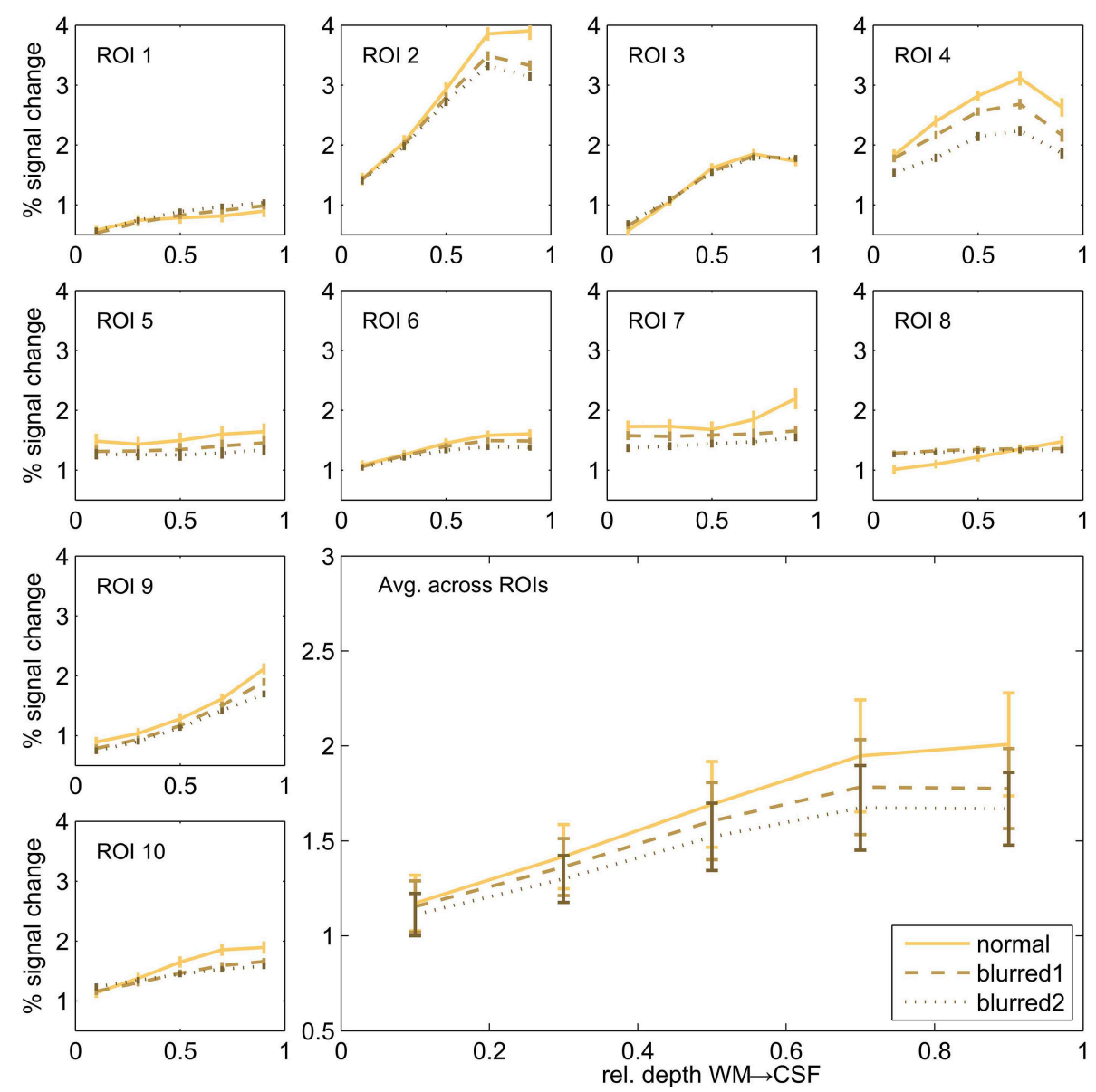

FIGURE 8 | Cortical depth profiles in individual regions. Single regions (small graphs) and their average (large graph, bottom right) of 2D SE-EPI 1. Original data is compared to blurred data of two different blurring kernel sizes.

by under-sampling and g-factor penalty. Additionally, although the FWHM of the 2D SE-EPI acquisitions was wider in the slice direction than expected $(1.3 \pm 0.2$ times the nominal slice thickness) the large overlap of neighboring slices in the multislice $2 \mathrm{D}$ acquisitions reduces the available magnetization because of saturation effects (Figure 3). Improved slice profiles could therefore improve both tSNR and point-spread.

The origin of the greater sensitivity of the 3D-GRASE sequence seems not to originate from differences in functional contrast (i.e., 3D-GRASE did not exhibit greater response amplitudes, as seen in the functional response profiles, which is also consistent with less contribution from pial vessels), but rather the higher sensitivity seems to originate from a more robust signal (i.e., less noise). The higher sensitivity permitted 3D-GRASE to detect more subtle BOLD signal changes than 2D SE-EPI, while larger responses were detected about equally.

\section{Contrast Mechanisms and Functional Specificity}

All acquisitions employed in this study elicit primarily $\mathrm{T}_{2}$ weighted contrast, with varying contributions from other mechanisms. As such, the bulk of the detected BOLD signal changes have absolute amplitudes below five percent, in agreement with the literature (Yacoub et al., 2005), however, it should be noted that differences in tasks, acquisition parameters (e.g., echo time, Koopmans et al., 2011), or sampling strategies may all result in differences in the observed functional signal changes. Contributions from $\mathrm{T}_{2}{ }^{*}\left(\mathrm{~T}_{2}^{\prime}\right)$ are not negligible due to the duration of the EPI echo trains, which was on the order of magnitude of the $\mathrm{T}_{2}{ }^{*}$ of the brain tissue. The $\mathrm{T}_{2}{ }^{*}$ contribution is expected to be higher in the 2D SE-EPI acquisitions than in 3D-GRASE because of the longer EPI echo trains and inplane partial Fourier acquisitions, as discussed in the section on the image PSF. The later (non-center) partitions in the 3DGRASE echo-train are sampled at a later effective TE than the nominal TE, which was matched with the TE of 2D SE-EPI 1. Therefore, the $\mathrm{T}_{2}$ contribution is higher in 3D-GRASE because later partitions experience more $\mathrm{T}_{2}$ weighting and additional $\mathrm{T}_{2}$ weighted stimulated echo weighting than the center partition (Goerke et al., 2007). In addition, blood vessels have a particularly sharp spatial profile (compared to smoother gray or white matter structures), which manifests itself in the k-space regions 

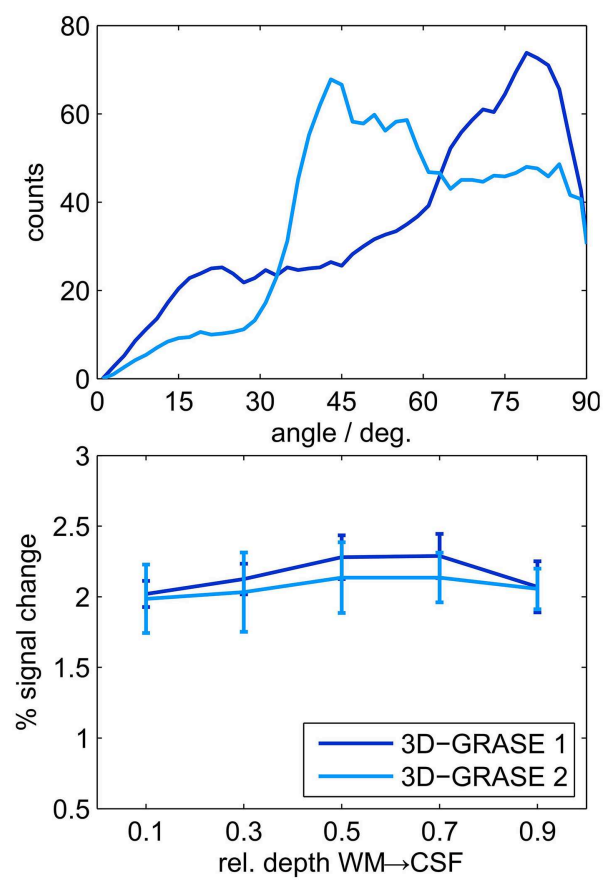

FIGURE 9 | Comparison of 3D-GRASE 1 and 3D-GRASE 2. Top: Histograms of angle between cortex normal and partition encoding direction of both, 3D-GRASE 1 (dark blue) and 3D-GRASE 2 (light blue), in degrees. Bottom: Mean cortical depth profiles. Error bars represent standard errors across regions.

corresponding to high spatial frequencies. High k-space lines of an EPI readout, in turn, experience the strongest $\mathrm{T}_{2}{ }^{*}$ weighting. Note that, in reduced FoV acquisitions, the same effect takes place. However, given the shorter EPI readout times, the high kspace lines are acquired closer to the spin-echo, thus reducing the effect size.

Finally, more diffusion weighting is aggregated along the readout train of 3D-GRASE due to the readout and crusher gradients. Moderate diffusion weighting has been shown to suppress macrovascular signals (Lee et al., 1999; Duong et al., 2003; Yacoub et al., 2003).

\section{References}

Auerbach, E. J., Heberlein, K., and Hu, X. (2002). "High-resolution T2 fMRI at high magnetic fields using PSIF," in Proceedings of the 10th Annual Meeting of ISMRM (Honolulu), 2345.

Bandettini, P. A., Wong, E. C., Hinks, R. S., Tikofsky, R. S., and Hyde, J. S. (1992). Time course EPI of human brain function during task activation. Magn. Reson. Med. 25, 390-397. doi: 10.1002/mrm.1910250220

Bandettini, P. A., Wong, E. C., Jesmanowicz, A., Hinks, R. S., and Hyde, J. S. (1994). Spin-echo and gradient-echo Epi of human brain activation using bold contrast - a comparative-study at 1.5 T. NMR Biomed. 7, 12-20. doi: 10.1002/nbm.1940070104

Barth, M., Meyer, H., Kannengiesser, S. A., Polimeni, J. R., Wald, L. L., and Norris, D. G. (2010). T2-weighted 3D fMRI using S2-SSFP at 7 tesla. Magn. Reson. Med. 63, 1015-1020. doi: $10.1002 / \mathrm{mrm} .22283$
In consequence of the aforementioned differences in contrast between 3D-GRASE and 2D SE-EPI, the cortical depth profiles differ such that 2D SE-EPI exhibits an increase of the signal change toward the pial surface, whereas 3D-GRASE remains mostly flat. Such increasing cortical depth profiles have previously been associated with reduced functional specificity in SE-EPI (Goense and Logothetis, 2006) and GE-EPI (Polimeni et al., 2010; De Martino et al., 2013), because they reflect $T_{2}{ }^{*}$ induced weighting of macrovasculature. We follow this line of argument and conclude that under the experimental conditions employed here, 3D-GRASE is less biased by large superficial effects as indicated by the layer profiles (Figure 7).

Our imaging PSF results raise the question of whether the observed difference in layer profiles is a mere consequence of the stronger blurring in the partition direction of 3D-GRASE. We show that this is not the case in two ways. First, we smoothed the 2D SE-EPI 1 data with anisotropic Gaussian filters exceeding the width of the PSF of 3D-GRASE along the partition direction. The characteristics of cortical depth profiles, and more specifically the increase toward the pial surface, are preserved (Figure 8). Second, when looking at the angle between the partition encoding direction and the direction of the cortical depth (Figure 9 top), on average there is not a bias of alignment between the partition direction and the grid direction (i.e., smaller angles between the directions). The curvature of the cortex prevents this from occurring and ultimately protects the cortical depth measures from the partition blurring of both 3D-GRASE 1 and 3D-GRASE 2, despite their orthogonal prescription. Further, the differences in angle distributions between the two acquisitions did not even elicit any significant difference in the layer profiles (Figure 9).

\section{Acknowledgments}

This study was supported by European Research Council (ERC) grant 269853, National Institute of Neurological Disorders and Stroke (NINDS) P30 NS076408, Biomedical Technology Resource Centers (BTRC), National Center for Research Resources (NCRR) P41 RR08079, and the National Institute of Biomedical Imaging and Bioengineering (NIBIB) P41 EB015894. FM was funded by NWO VIDI (grant 864-13-012).

Bernstein, M. A., King, K. F., and Zhou, X. J. (2004). Handbook of MRI Pulse Sequences. Oxford: Elsevier.

Bieri, O., and Scheffler, K. (2007). Effect of diffusion in inhomogeneous magnetic fields on balanced steady-state free precession. NMR Biomed. 20, 1-10. doi: 10.1002/nbm.1079

Boxerman, J. L., Hamberg, L. M., Rosen, B. R., and Weisskoff, R. M. (1995). $\mathrm{Mr}$ contrast due to intravascular magnetic-susceptibility perturbations. Magn. Reson. Med. 34, 555-566. doi: 10.1002/mrm.1910340412

Boyacioglu, R., Schulz, J., Muller, N. C., Koopmans, P. J., Barth, M., and Norris, D. G. (2014). Whole brain, high resolution multiband spin-echo EPI fMRI at 7T: a comparison with gradient-echo EPI using a color-word Stroop task. Neuroimage 97, 142-150. doi: 10.1016/j.neuroimage.2014.04.011

Chung, J. Y., In, M. H., Oh, S. H., Zaitsev, M., Speck, O., and Cho, Z. H. (2011). An improved PSF mapping method for EPI distortion correction in human brain at ultra high field (7T). MAGMA 24, 179-190. doi: 10.1007/s10334-011-0251-1 
Constable, R. T., and Gore, J. C. (1992). The loss of small objects in variable TE imaging: implications for FSE, RARE, and EPI. Magn. Reson. Med. 28, 9-24. doi: $10.1002 / \mathrm{mrm} .1910280103$

Constable, R. T., Kennan, R. P., Puce, A., McCarthy, G., and Gore, J. C. (1994). Functional NMR imaging using fast spin echo at 1.5 T. Magn. Reson. Med. 31, 686-690. doi: 10.1002/mrm.1910310617

Cox, E. F., and Gowland, P. A. (2010). Simultaneous quantification of T2 and T'2 using a combined gradient echo-spin echo sequence at ultrahigh field. Magn. Reson. Med. 64, 1440-1445. doi: 10.1002/mrm.22522

Deistung, A., Rauscher, A., Sedlacik, J., Stadler, J., Witoszynskyj, S., and Reichenbach, J. R. (2008). Susceptibility weighted imaging at ultra high magnetic field strengths: theoretical considerations and experimental results. Magn. Reson. Med. 60, 1155-1168. doi: 10.1002/mrm.21754

De Martino, F., Zimmermann, J., Muckli, L., Ugurbil, K., Yacoub, E., and Goebel, R. (2013). Cortical depth dependent functional responses in humans at 7T: improved specificity with 3D GRASE. PLoS ONE 8:e60514. doi: 10.1371/journal.pone.0060514

Duong, T. Q., Yacoub, E., Adriany, G., Hu, X., Ugurbil, K., and Kim, S. G. (2003). Microvascular BOLD contribution at 4 and $7 \mathrm{~T}$ in the human brain: gradientecho and spin-echo fMRI with suppression of blood effects. Magn. Reson. Med. 49, 1019-1027. doi: 10.1002/mrm.10472

Duong, T. Q., Yacoub, E., Adriany, G., Hu, X., Ugurbil, K., Vaughan, J. T., et al. (2002). High-resolution, spin-echo BOLD, and CBF fMRI at 4 and 7 T. Magn. Reson. Med. 48, 589-593. doi: 10.1002/mrm.10252

Edelstein, W. A., Glover, G. H., Hardy, C. J., and Redington, R. W. (1986). The intrinsic signal-to-noise ratio in NMR imaging. Magn. Reson. Med. 3, 604-618. doi: $10.1002 / \mathrm{mrm} .1910030413$

Feinberg, D. A., Hale, J. D., Watts, J. C., Kaufman, L., and Mark, A. (1986). Halving MR imaging time by conjugation: demonstration at $3.5 \mathrm{kG}$. Radiology 161, 527-531. doi: 10.1148/radiology.161.2.3763926

Feinberg, D. A., Harel, N., Ramanna, S., Ugurbil, K., and Yacoub, E. (2008). "Submillimeter single-shot 3D GRASE with inner volume selection for T2 weighted fMRI applications at 7 Tesla," in 16th Annual Meeting International Society for Magnetic Resonance in Medicine (Toronto, ON), 2373.

Finsterbusch, J. (2010). Fast-spin-echo imaging of inner fields-of-view with 2D-selective RF excitations. J. Magn. Reson. Imaging 31, 1530-1537. doi: 10.1002/jmri.22196

Finsterbusch, J. (2013). Functional neuroimaging of inner fields-of-view with 2D-selective RF excitations. Magn. Reson. Imaging 31, 1228-1235. doi: 10.1016/j.mri.2013.03.005

Goa, P. E., Koopmans, P. J., Poser, B. A., Barth, M., and Norris, D. G. (2014). BOLD fMRI signal characteristics of S1- and S2-SSFP at 7 Tesla. Front. Neurosci. 8:49. doi: 10.3389/fnins.2014.00049

Goense, J. B., and Logothetis, N. K. (2006). Laminar specificity in monkey V1 using high-resolution SE-fMRI. Magn. Reson. Imaging 24, 381-392. doi: 10.1016/j.mri.2005.12.032

Goense, J., Merkle, H., and Logothetis, N. K. (2012). High-resolution fMRI reveals laminar differences in neurovascular coupling between positive and negative BOLD responses. Neuron 76, 629-639. doi: 10.1016/j.neuron.2012. 09.019

Goerke, U., van de Moortele, P. F., and Ugurbil, K. (2007). Enhanced relative BOLD signal changes in T(2)-weighted stimulated echoes. Magn. Reson. Med. 58, 754-762. doi: 10.1002/mrm.21369

Gomori, J., Holland, G., Grossman, R., Gefter, W., and Lenkinski, R. (1988). Fat suppression by section-select gradient reversal on spinecho MR imaging. Work in progress. Radiology 168, 493-495. doi: 10.1148/radiology.168.2.3393670

Harel, N., Lin, J., Moeller, S., Ugurbil, K., and Yacoub, E. (2006). Combined imaging-histological study of cortical laminar specificity of fMRI signals. Neuroimage 29, 879-887. doi: 10.1016/j.neuroimage.2005.08.016

Harmer, J., Sanchez-Panchuelo, R. M., Bowtell, R., and Francis, S. T. (2012). Spatial location and strength of BOLD activation in high-spatial-resolution fMRI of the motor cortex: a comparison of spin echo and gradient echo fMRI at 7 T. NMR Biomed. 25, 717-725. doi: 10.1002/nbm.1783

Heidemann, R. M., Ivanov, D., Trampel, R., Fasano, F., Meyer, H., Pfeuffer, J., et al. (2012). Isotropic submillimeter fMRI in the human brain at $7 \mathrm{~T}$ : combining reduced field-of-view imaging and partially parallel acquisitions. Magn. Reson. Med. 68, 1506-1516. doi: 10.1002/mrm.24156
Hennig, J., Nauerth, A., and Friedburg, H. (1986). RARE imaging: a fast imaging method for clinical MR. Magn. Reson. Med. 3, 823-833. doi: 10.1002/mrm.1910030602

Huber, L., Goense, J., Kennerley, A. J., Trampel, R., Guidi, M., Reimer, E., et al. (2015). Cortical lamina-dependent blood volume changes in human brain at 7 T. Neuroimage 107, 23-33. doi: 10.1016/j.neuroimage.2014.11.046

Ivanov, D., Schafer, A., Streicher, M. N., Heidemann, R. M., Trampel, R., and Turner, R. (2010). A simple low-SAR technique for chemical-shift selection with high-field spin-echo imaging. Magn. Reson. Med. 64, 319-326. doi: $10.1002 / \mathrm{mrm} .22518$

Jin, T., and Kim, S.-G. (2008). Cortical layer-dependent dynamic blood oxygenation, cerebral blood flow and cerebral blood volume responses during visual stimulation. Neuroimage 43, 1-9. doi: 10.1016/j.neuroimage.2008.06.029

Jones, S. E., Buchbinder, B. R., and Aharon, I. (2000). Three-dimensional mapping of cortical thickness using Laplace's equation. Hum. Brain Mapp. 11, 12-32. doi: 10.1002/1097-0193(200009)11:1<12::AID-HBM20>3.0.CO;2-K

Koning, W., Bluemink, J. J., Langenhuizen, E. A., Raaijmakers, A. J., Andreychenko, A., van den Berg, C. A., et al. (2013). High-resolution MRI of the carotid arteries using a leaky waveguide transmitter and a high-density receive array at 7 T. Magn. Reson. Med. 69, 1186-1193. doi: 10.1002/mrm.24345

Koopmans, P. J., Barth, M., Orzada, S., and Norris, D. G. (2011). Multi-echo fMRI of the cortical laminae in humans at 7 T. Neuroimage 56, 1276-1285. doi: 10.1016/j.neuroimage.2011.02.042

Kwong, K. K., Belliveau, J. W., Chesler, D. A., Goldberg, I. E., Weisskoff, R. M., Poncelet, B. P., et al. (1992). Dynamic magnetic resonance imaging of human brain activity during primary sensory stimulation. Proc. Natl. Acad. Sci. U.S.A. 89, 5675-5679. doi: 10.1073/pnas.89.12.5675

Lee, S.-P., Silva, A. C., Ugurbil, K., and Kim, S.-G. (1999). Diffusion-weighted spinecho fMRI at 9.4 T: microvascular/tissue contribution to BOLD signal changes. Magn. Reson. Med. 42, 919-928.

Mansfield, P. (1977). Multi-planar image formation using NMR spin echoes. J. Phys. C Solid State Phys. 10, L55. doi: 10.1088/0022-3719/10/3/004

Miller, K. L., and Jezzard, P. (2008). Modeling SSFP functional MRI contrast in the brain. Magn. Reson. Med. 60, 661-673. doi: 10.1002/mrm.21690

Mugler, J. P. III, Epstein, F. H., and Brookeman, J. R. (1992). Shaping the signal response during the approach to steady state in three-dimensional magnetization-prepared rapid gradient-echo imaging using variable flip angles. Magn. Reson. Med. 28, 165-185. doi: 10.1002/mrm.1910280202

Murphy, K., Bodurka, J., and Bandettini, P. A. (2007). How long to scan? The relationship between $\mathrm{fMRI}$ temporal signal to noise ratio and necessary scan duration. Neuroimage 34, 565-574. doi: 10.1016/j.neuroimage.2006.09.032

Norris, D. G. (2012). Spin-echo fMRI: the poor relation? Neuroimage 62, 1109-1115. doi: 10.1016/j.neuroimage.2012.01.003

Ogawa, S., Lee, T. M., Kay, A. R., and Tank, D. W. (1990). Brain magnetic resonance imaging with contrast dependent on blood oxygenation. Proc. Natl. Acad. Sci. U.S.A. 87, 9868-9872. doi: 10.1073/pnas.87.24.9868

Ogawa, S., Tank, D. W., Menon, R., Ellermann, J. M., Kim, S. G., Merkle, H., et al. (1992). Intrinsic signal changes accompanying sensory stimulation: functional brain mapping with magnetic resonance imaging. Proc. Natl. Acad. Sci. U.S.A. 89, 5951-5955. doi: 10.1073/pnas.89.13.5951

Olman, C. A., Harel, N., Feinberg, D. A., He, S., Zhang, P., Ugurbil, K., et al. (2012). Layer-specific fMRI reflects different neuronal computations at different depths in human V1. PLoS ONE 7:e32536. doi: 10.1371/journal.pone.0032536

Oshio, K., and Feinberg, D. A. (1991). GRASE (Gradient- and spin-echo) imaging: a novel fast MRI technique. Magn. Reson. Med. 20, 344-349. doi: 10.1002/mrm.1910200219

Panchuelo, R. M. S., Schluppeck, D., Harmer, J., Bowtell, R., and Francis, S. (2015). Assessing the Spatial Precision of SE and GE-BOLD Contrast at 7 Tesla. Brain Topogr. 28, 62-65. doi: 10.1007/s10548-014-0420-4

Park, H. W., Kim, D. J., and Cho, Z. H. (1987). Gradient reversal technique and its applications to chemical-shift-related NMR imaging. Magn. Reson. Med. 4, 526-536. doi: 10.1002/mrm.1910040604

Park, J., Mugler, J. P. III, Horger, W., and Kiefer, B. (2007). Optimized T1weighted contrast for single-slab 3D turbo spin-echo imaging with long echo trains: application to whole-brain imaging. Magn. Reson. Med. 58, 982-992. doi: 10.1002/mrm. 21386

Pauly, J., Nishimura, D., and Macovski, A. (1989). A k-space analysis of small-tipangle excitation. J. Magn. Reson. 81, 43-56. doi: 10.1016/0022-2364(89)90265-5 
Peters, A. M., Brookes, M. J., Hoogenraad, F. G., Gowland, P. A., Francis, S. T., Morris, P. G., et al. (2007). T2* measurements in human brain at 1.5, 3 and $7 \mathrm{~T}$. Magn. Reson. Imaging 25, 748-753. doi: 10.1016/j.mri.2007.02.014

Pfeuffer, J., van de Moortele, P.-F., Yacoub, E., Shmuel, A., Adriany, G., Andersen, P., et al. (2002). Zoomed functional imaging in the human brain at 7 Tesla with simultaneous high spatial and high temporal resolution. Neuroimage 17, 272-286. doi: 10.1006/nimg.2002.1103

Pflugfelder, D., Vahedipour, K., Uludag, K., Shah, N. J., and Stocker, T. (2011). On the numerically predicted spatial BOLD fMRI specificity for spin echo sequences. Magn. Reson. Imaging 29, 1195-1204. doi: 10.1016/j.mri.2011.07.015

Polimeni, J. R., Fischl, B., Greve, D. N., and Wald, L. L. (2010). Laminar analysis of 7T BOLD using an imposed spatial activation pattern in human V1. Neuroimage 52, 1334-1346. doi: 10.1016/j.neuroimage.2010.05.005

Poser, B. A., and Norris, D. G. (2007). Fast spin echo sequences for BOLD functional MRI. MAGMA 20, 11-17. doi: 10.1007/s10334-006-0063-x

Robson, M. D., Gore, J. C., and Constable, R. T. (1997). Measurement of the point spread function in MRI using constant time imaging. Magn. Reson. Med. 38, 733-740. doi: 10.1002/mrm.1910380509

Shmuel, A., Yacoub, E., Chaimow, D., Logothetis, N. K., and Ugurbil, K. (2007). Spatio-temporal point-spread function of fMRI signal in human gray matter at 7 Tesla. Neuroimage 35, 539-552. doi: 10.1016/j.neuroimage.2006.12.030

Triantafyllou, C., Hoge, R. D., Krueger, G., Wiggins, C. J., Potthast, A., Wiggins, G, C., et al. (2005). Comparison of physiological noise at $1.5 \mathrm{~T}, 3 \mathrm{~T}$ and $7 \mathrm{~T}$ and optimization of fMRI acquisition parameters. Neuroimage 26, 243-250. doi: 10.1016/j.neuroimage.2005.01.007

Uludag, K., Muller-Bierl, B., and Ugurbil, K. (2009). An integrative model for neuronal activity-induced signal changes for gradient and spin echo functional imaging. Neuroimage 48, 150-165. doi: 10.1016/j.neuroimage.2009.05.051

van de Moortele, P. F., Auerbach, E. J., Olman, C., Yacoub, E., Ugurbil, K., and Moeller, S. (2009). T1 weighted brain images at 7 Tesla unbiased for Proton Density, $2^{*}$ contrast and RF coil receive B1 sensitivity with simultaneous vessel visualization. Neuroimage 46, 432-446. doi: 10.1016/j.neuroimage.2009.02.009

Volk, A., Tiffon, B., Mispelter, J., and Lhoste, J.-M. (1987). Chemical shift-specific slice selection. A new method for chemical shift imaging at high magnetic field. J. Magn. Reson. 71, 168-174.

Wargo, C. J., and Gore, J. C. (2013). Localized high-resolution DTI of the human midbrain using single-shot EPI, parallel imaging, and outer-volume suppression at 7 T. Magn. Reson. Imaging 31, 810-819. doi: 10.1016/j.mri.2013.01.013

Yacoub, E., Duong, T. Q., van de Moortele, P. F., Lindquist, M., Adriany, G., Kim, S. G., et al. (2003). Spin-echo fMRI in humans using high spatial resolutions and high magnetic fields. Magn. Reson. Med. 49, 655-664. doi: 10.1002/mrm.10433

Yacoub, E., Harel, N., and Ugurbil, K. (2008). High-field fMRI unveils orientation columns in humans. Proc. Natl. Acad. Sci. U.S.A. 105, 10607-10612. doi: 10.1073/pnas.0804110105

Yacoub, E., Shmuel, A., Logothetis, N., and Ugurbil, K. (2007). Robust detection of ocular dominance columns in humans using Hahn Spin Echo BOLD functional MRI at 7 Tesla. Neuroimage 37, 1161-1177. doi: 10.1016/j.neuroimage.2007.05.020

Yacoub, E., van de Moortele, P. F., Shmuel, A., and Ugurbil, K. (2005). Signal and noise characteristics of Hahn SE and GE BOLD fMRI at $7 \mathrm{~T}$ in humans. Neuroimage 24, 738-750. doi: 10.1016/j.neuroimage.2004.09.002

Zaitsev, M., Hennig, J., and Speck, O. (2004). Point spread function mapping with parallel imaging techniques and high acceleration factors: fast, robust, and flexible method for echo-planar imaging distortion correction. Magn. Reson. Med. 52, 1156-1166. doi: 10.1002/mrm.20261

Zeng, H., and Constable, R. T. (2002). Image distortion correction in EPI: comparison of field mapping with point spread function mapping. Magn. Reson. Med. 48, 137-146. doi: 10.1002/mrm.10200

Zimmermann, J., Goebel, R., De Martino, F., van de Moortele, P. F., Feinberg, D., Adriany, G., et al. (2011). Mapping the organization of axis of motion selective features in human area MT using high-field fMRI. PLOS ONE 6:e28716. doi: 10.1371/journal.pone.0028716

Conflict of Interest Statement: The authors declare that the research was conducted in the absence of any commercial or financial relationships that could be construed as a potential conflict of interest.

Copyright (c) 2015 Kemper, De Martino, Vu, Poser, Feinberg, Goebel and Yacoub. This is an open-access article distributed under the terms of the Creative Commons Attribution License (CC BY). The use, distribution or reproduction in other forums is permitted, provided the original author(s) or licensor are credited and that the original publication in this journal is cited, in accordance with accepted academic practice. No use, distribution or reproduction is permitted which does not comply with these terms. 